\title{
Optical Potentials: Microscopic vs. Phenomenological Approaches
}

\author{
Paolo Finelli ${ }^{1}$, Matteo Vorabbi $^{2}$, and Carlotta Giusti $^{3}$ \\ ${ }^{1}$ Astronomy and Physics Department, University of Bologna (Italy) and INFN section of Bologna (Italy) \\ ${ }^{2}$ TRIUMF, Vancouver (Canada) \\ ${ }^{3}$ Physics Department, University of Pavia (Italy) and INFN section of Pavia (Italy)
}

\begin{abstract}
In this work we study the performances of our microscopic optical potential $[1,2]$, derived from nucleon-nucleon chiral potentials at fifth order $\left(\mathrm{N}^{4} \mathrm{LO}\right)$, in comparison with those of a successful non-relativistic phenomenological optical potential in the description of elastic proton scattering data on tin and lead isotopes at energies around and above $200 \mathrm{MeV}$. Our results indicate that microscopic optical potentials derived from nucleon-nucleon chiral potentials at $\mathrm{N}^{4} \mathrm{LO}$ can provide reliable predictions for observables of stable and exotic nuclei, even at energies where the robustness of the chiral expansion starts to be questionable.
\end{abstract}

\section{Introduction}

In Ref. [1] we constructed a microscopic optical potential (OP) for elastic proton-nucleus (pA) scattering starting from nucleon-nucleon $(\mathrm{NN})$ chiral potentials derived up to $\mathrm{N}^{3} \mathrm{LO}$ in the chiral expansion and we studied the chiral convergence of the $\mathrm{NN}$ potential in reproducing the pA scattering observables. The OP was obtained at the first-order term within the spectator expansion of the nonrelativistic multiple scattering theory and adopting the impulse approximation and the optimum factorization approximation.

In a subsequent work [2] we adopted the same model to obtain the OP and we studied the chiral convergence of a new generation of $\mathrm{NN}$ chiral interactions derived up to $\mathrm{N}^{4} \mathrm{LO}$.

In this contribution we show a sample of our latest work [3] about the predictive power of our microscopic OP derived in Ref. [2] from different chiral potentials at $\mathrm{N}^{4} \mathrm{LO}$ and of the successful phenomenological OP (KD) of Ref. [12] in comparison with available data for the observables of elastic proton scattering on different isotopic chains. Results are presented for several proton energies around and above $200 \mathrm{MeV}$, with the aim to test the upper energy limit of applicability of our OP before the chiral expansion scheme breaks down.

\section{Theory}

The theoretical justification for the description of the $\mathrm{pA}$ optical potential in terms of the microscopical NN interaction has been addressed for the first time by Watson et al. [4] and then formalized by Kerman et al. (KMT) [5], where the so-called multiple scattering approach to the $\mathrm{pA}$ optical potential is expressed by a series expansion of the free NN scattering amplitudes.
In Refs. [1, 2] a microscopic OP $V_{o p t}$ was obtained at the first-order term within the spectator expansion of the nonrelativistic multiple scattering theory, corresponding to the single-scattering approximation. The impulse approximation was adopted, where nuclear binding forces on the interacting target nucleon are neglected, as well as the optimum factorization approximation, where the two basic ingredients of the calculations, i.e. the nuclear density and the NN $t$ matrix, are factorized. We refer the reader to Refs. [1, 2] for all relevant details and an exhaustive bibliography. In the momentum space, the factorized $V_{o p t}$ is obtained as

$$
V_{o p t}(\mathbf{q}, \mathbf{K} ; \omega) \sim \sum_{N=n, p} t_{p N}\left[\mathbf{q}, \frac{A+1}{A} \mathbf{K} ; \omega\right] \rho_{N}(q),
$$

where $t_{p N}$ represents the proton-proton (pp) and protonneutron (pn) free $t$ matrix evaluated at a fixed energy $\omega$, $\rho_{N}$ the neutron and proton profile density, and the incoming and outgoing projectile momenta $\mathbf{k}$ and $\mathbf{k}^{\prime}$ are conveniently expressed by the variables $\mathbf{q} \equiv \mathbf{k}^{\prime}-\mathbf{k}$ and $\mathbf{K} \equiv \frac{1}{2}\left(\mathbf{k}^{\prime}+\mathbf{k}\right)($ see Sect. II of Ref. [1]).

For the neutron and proton densities of the target nucleus we use as in Refs. [1,2] a Relativistic Mean-Field (RMF) description [6], which has been quite successful in the description of ground state and excited state properties of finite nuclei, in particular in a Density Dependent Meson Exchange (DDME) version, where the couplings between mesonic and baryonic fields are assumed as functions of the density itself [7].

For the NN interaction we use here two different versions of the chiral potentials at fifth order $\left(\mathrm{N}^{4} \mathrm{LO}\right)$ recently derived by Epelbaum, Krebs, and Meißner (EKM) [8, 9] and Entem, Machleidt, and Nosyk (EMN) [10, 11]. As explained in Ref. [2], the two versions of the chiral $\mathrm{N}^{4} \mathrm{LO}$ potentials have significant differences concerning the renormalization procedures and we follow the same 


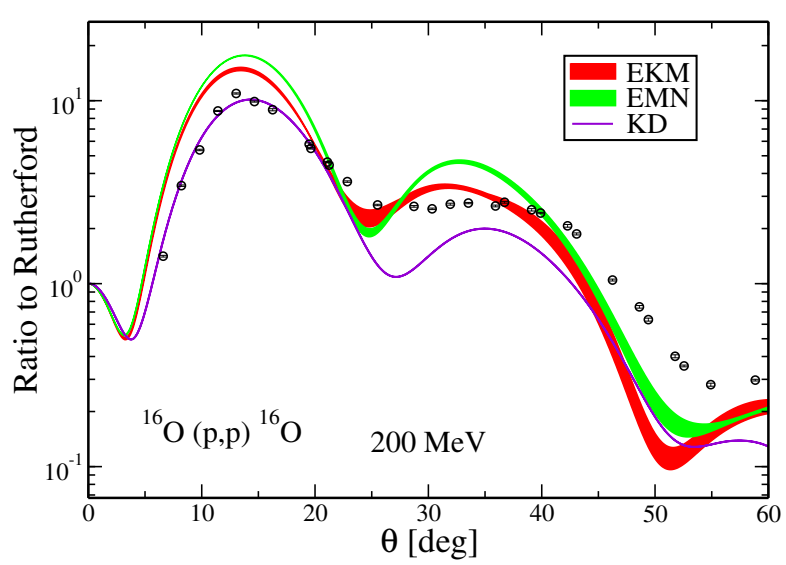

Figure (1) Ratio of the differential cross section to the Rutherford cross section as a function of the center-ofmass scattering angle $\theta$ for elastic proton scattering off ${ }^{16} \mathrm{O}$. Calculations are performed at $E=200 \mathrm{MeV}$ (laboratory energy) with the microscopic OPs derived from the EKM [8, 9](EKM, red band) and EMN [10, 11] (EMN, green band) $\mathrm{NN}$ chiral potentials at $\mathrm{N}^{4} \mathrm{LO}$ and with the phenomenological global OP of Ref. [12] (KD, violet line). The interpretation of the bands is explained in the text. Experimental data from Refs. [13, 14].

prescriptions adopted there. The strategy followed for the EKM potentials $[8,9]$ consists in a coordinate space regularization for the long-range contributions $V_{\text {long }}(\boldsymbol{r})$, by the introduction of $f\left(\frac{r}{R}\right)=\left(1-\exp \left(-\frac{r^{2}}{R^{2}}\right)\right)^{n}$, and a conventional momentum space regularization for the contact (short-range) terms, with a cutoff $\Lambda=2 R^{-1}$. Five choices of $R$ are available: $0.8,0.9,1.0,1.1$, and $1.2 \mathrm{fm}$, leading to five different potentials.

On the other hand, for the EMN potentials, a slightly more conventional approach was pursued $[10,11]$. A spectral function regularization, with a cutoff $\tilde{\Lambda} \simeq 700$ $\mathrm{MeV}$, was employed to regularize the loop contributions and a conventional regulator function, with $\Lambda=450,500$, and $550 \mathrm{MeV}$, to deal with divergences in the LippmannSchwinger equation. For all details we refer the reader to Ref. [2].

Since the goal of the present work is to test the predictive power of our microscopic OP in comparison with available experimental data, it is definitely interesting to show the uncertainties on the predictions produced by NN chiral potentials obtained with different values of the regularization parameters. For this purpose, all calculations have been performed with three of the EKM [8, 9] potentials, corresponding to $R=0.8,0.9$, and $1.0 \mathrm{fm}$, and with two of the EMN $[10,11]$ potentials, corresponding to $\Lambda=500$ and $550 \mathrm{MeV}$.

In all the next figures the bands give the differences produced by changing $R$ for EKM (red bands) and $\Lambda$ for EMN (green bands). Thus the bands have here a different meaning than in Ref. [2], where the EKM and EMN $\mathrm{NN}$ chiral potentials at $\mathrm{N}^{4} \mathrm{LO}$ were also used. The aim of Ref. [2] was to investigate the convergence and to assess the theoretical errors associated with the truncation of the chiral expansion and the bands were given to investigate these issues.

We also showed in Ref. [2] that EKM calculations based on different values of $R$ are quite close and consistent with each other (although, as remarked in Ref. [8], larger values of $\mathrm{R}$ are probably less accurate due to a larger influence of cutoff artifacts). The same assumption can be made about the EMN potentials: changing the cutoffs does not lead to sizeable differences in the $\chi^{2} /$ datum (see Tab.VIII in Ref. [11]) and it is safe to perform calculations with only two potentials. Because we want to explore elastic scattering at energies around and above $200 \mathrm{MeV}$, we exclude the EKM potentials with $R=1.1$ and $1.2 \mathrm{fm}$ and the EMN potential with $\Lambda=450 \mathrm{MeV}$.

In Ref. [1] we compared the results obtained with different versions of EKM and EMN chiral potentials at $\mathrm{N}^{4} \mathrm{LO}$ for the pp and pn Wolfenstein amplitudes and for the scattering observables of elastic proton scattering off ${ }^{12} \mathrm{C},{ }^{16} \mathrm{O}$, and ${ }^{40} \mathrm{Ca}$ nuclei at an incident proton energy of $E=200 \mathrm{MeV}$.

We show in Fig. 1 the ratio of the differential cross section to the Rutherford cross section for elastic proton scattering off ${ }^{16} \mathrm{O}$ at $E=200 \mathrm{MeV}$. The results obtained with the EKM and EMN potentials and with the KD optical potential are compared with the experimental data taken from Refs. [13, 14]. The EKM and EMN results for the differential cross section give a reasonable, although not perfect, agreement with data. The experimental ratio is slightly overestimated at lower angles and somewhat underestimated for $\theta \geq 50^{\circ}$. The differences between the EKM and EMN results are small and not crucial, EKM gives a smaller cross section around the maxima and therefore a somewhat better agreement with the data in this region. The bands, representing the uncertainties on the regularization of the NN chiral potentials, are generally small and not influential for the comparison with data. The KD result gives a good description of the experimental cross section for $\theta \leq 20^{\circ}$ and underpredicts the data for larger angles.

The results for $\mathrm{Sn}$ isotopes at $295 \mathrm{MeV}$ and for ${ }^{120} \mathrm{Sn}$ at $200 \mathrm{MeV}$ are displayed in the upper panel of Fig. 2. In this case all the OPs give qualitatively similar results and a reasonable agreement with data, in particular, for $\theta \leq 20^{\circ}$. The agreement generally declines for larger angles. KD gives a better description of ${ }^{120} \mathrm{Sn}$ data at $200 \mathrm{MeV}$, where the EKM and EMN results are a bit larger than the data at the maxima and a bit lower at the minima. We note that ${ }^{120} \mathrm{Sn}$ is included in the experimental database for the $\mathrm{KD}$ potential for proton energies up to $160 \mathrm{MeV}$. At 295 $\mathrm{MeV}$, the microscopic OP gives, in general, a slightly better agreement with the data than KD for all the tin isotopes shown in the figure.

The results for $\mathrm{Pb}$ isotopes at $295 \mathrm{MeV}$ and for ${ }^{208} \mathrm{~Pb}$ data at $200 \mathrm{MeV}$ are displayed in lower panel of Fig. 2. Also in this case the experimental cross section at 200 $\mathrm{MeV}$ is well described by $\mathrm{KD}$, the agreement is better than with the microscopic OP. The experimental database for KD includes ${ }^{208} \mathrm{~Pb}$ up to $200 \mathrm{MeV}$. At $295 \mathrm{MeV}$ a better agreement with data is generally given by the EKM and EMN results, in particular by EMN: for all the three iso- 

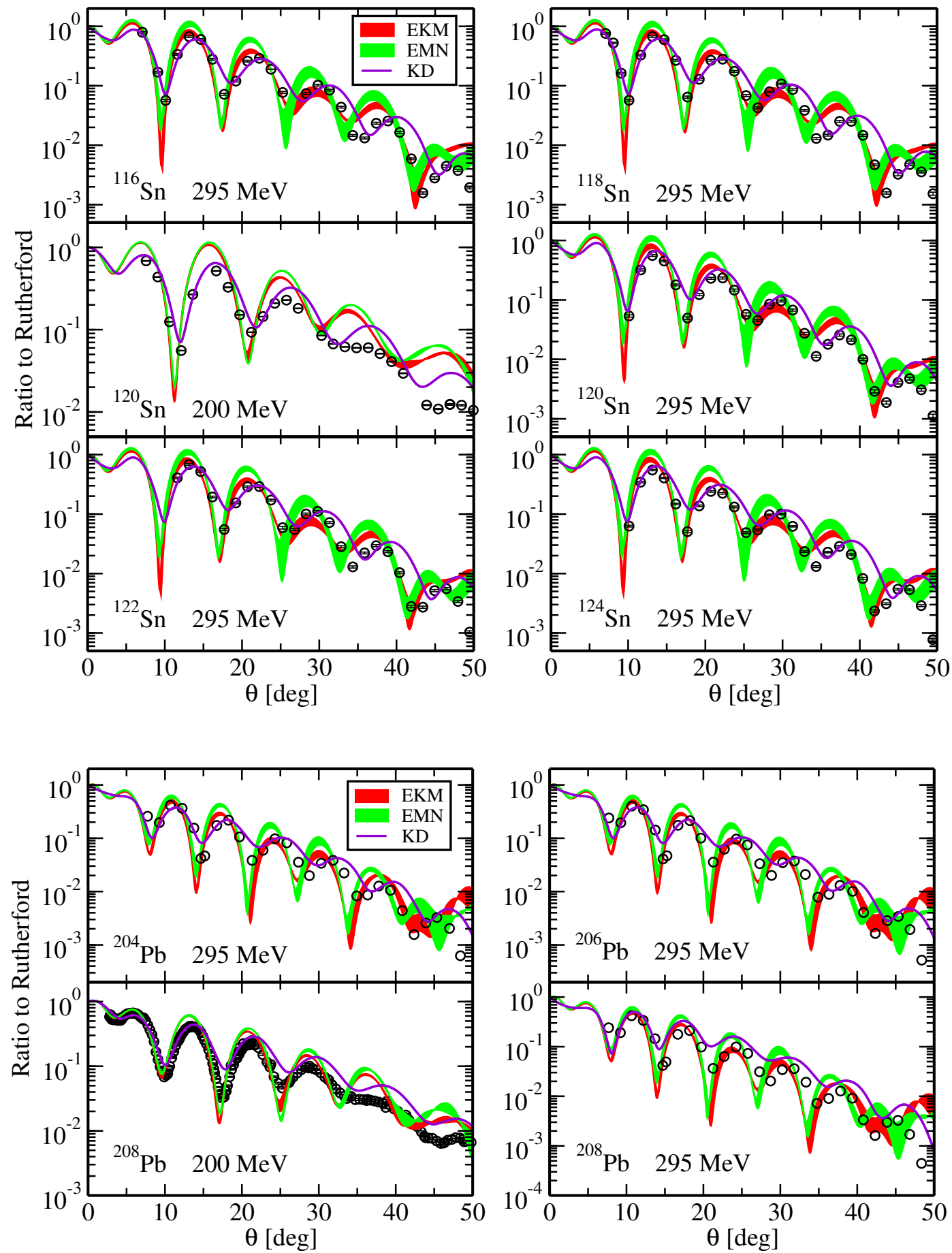

Figure (2) (Upper panel) The same as in Fig. 1 for $\mathrm{Sn}$ isotopes: ${ }^{120} \mathrm{Sn}$ at $E=200 \mathrm{MeV}$ and ${ }^{116,118,120,122,124} \mathrm{Sn}$ at $E=295 \mathrm{MeV}$. Experimental data from Refs. [13, 14]. (Lower panel) The same as in Fig. 1 for Pb isotopes: ${ }^{208} \mathrm{~Pb}$ at $E=200 \mathrm{MeV}$ and ${ }^{204,206,208} \mathrm{~Pb}$ at $E=295 \mathrm{MeV}$. Experimental data from Refs. [13, 14].

topes considered, the two results practically overlap for $\theta \leq 20^{\circ}$, where they are also very close to the KD result, then they start to separate and the EMN result is a bit larger than the EKM one and in better agreement with data. We point out that the uncertainty bands, that are generally narrow, in this case become larger increasing the scattering angle, when also the agreement with data declines.
The results that we have shown till now indicate that, in comparison with the phenomenological KD potential, our microscopic OP, in spite of the approximations made to derived it, has a comparable and in some cases even better predictive power in the description of the cross sections on the isotopic chains and energy range here considered. $\mathrm{KD}$ is able to give a better and excellent description of data in specific situations, in particular, for data included 
in the experimental database used to obtain the parameters of the phenomenological KD potential and at the lower energies considered. Our microscopic OP is able to give a similar and more homogeneous description of data for all the nuclei of an isotopic chain and, for energies above 200 $\mathrm{MeV}$ it gives, in general, a better agreement with data than the phenomenological KD potential.

\section{References}

[1] M. Vorabbi, P. Finelli, and C. Giusti, Phys. Rev. C 93, 034619 (2016)

[2] M. Vorabbi, P. Finelli, and C. Giusti, Phys. Rev. C 96, 044001 (2017)

[3] M. Vorabbi, P. Finelli, and C. Giusti, Phys. Rev. C 98, 064602 (2018)

[4] W. B. Riesenfeld and K. M. Watson, Phys. Rev. 102, 1157 (1956)
[5] A. K. Kerman, H. McManus, and R. M. Thaler, Annals Phys. 8, 551 (1959)

[6] T. Niksic, N. Paar, D. Vretenar, and P. Ring, Computer Physics Communications 185, 1808 (2014)

[7] T. Niksic, D. Vretenar, P. Finelli, and P. Ring, Phys. Rev. C 66, 024306 (2002)

[8] 'E. Epelbaum, H. Krebs, and U. G. Meissner, Phys. Rev. Lett. 115122301 (2015)

[9] E. Epelbaum, H. Krebs, and U. G. Meissner, Eur. Phys. J. A 5153 (2015)

[10] D. R. Entem, N. Kaiser, R. Machleidt, and Y. Nosyk, Phys. Rev. C 91014002 (2015)

[11] D. R. Entem, R. Machleidt, Y. Nosyk, Phys. Rev. C96 024004 (2017)

[12] A. J. Koning and J. P. Delaroche, Nucl. Phys. A 713, 231 (2003)

[13] http://www.nndc.bnl.gov/exfor/exfor.htm

[14] http://www.physics.umd.edu/enp/jjkelly/ 